\title{
Direct Measurement of a Mode-Converted Electron Bernstein Wave in the Internal Coil Device Mini-RT
}

\author{
Eiichi YATSUKA, Daisuke SAKATA, Kiyotake KINJO, Junji MORIKAWA ${ }^{1)}$ and Yuichi OGAWA ${ }^{1)}$ \\ Graduate School of Frontier Sciences, University of Tokyo, Kashiwa 277-8561, Japan \\ ${ }^{1)}$ High Temperature Plasma Center, University of Tokyo, Kashiwa 277-8568, Japan
}

(Received 21 December 2007 / Accepted 30 January 2008)

\begin{abstract}
An electric field measurement system in the Electron Cyclotron Range of Frequency (ECRF) has been developed for the direct detection of mode-converted Electron Bernstein Waves (EBWs), where a low-power ECRF wave $\left(1-2.1 \mathrm{GHz}, 10 \mathrm{~W}\right.$ ) is injected into overdense plasmas (typical peak density is $7 \times 10^{16} \mathrm{~m}^{-3}$ ), and the ECRF electric field is directly measured using a small monopole antenna (an element size of $5 \mathrm{~mm}$ ) inserted into the confined plasma. A density gradient is an important parameter for mode conversion to EBW. In the internal coil device, Mini-RT, a steep density gradient (e.g., $L_{\mathrm{n}} \sim 5 \mathrm{~cm}$ ) is available at the plasma surface. We have detected short-wavelength waves with refractive indices of $\sim 10$ or more around the electron cyclotron harmonic resonance layers, and confirmed a phase reversal of an ECRF electric field at the same locations. Since the EBW is a backward wave with a short wavelength, experimental results suggest the direct measurement of the mode-converted EBW in plasma.
\end{abstract}

(C) 2008 The Japan Society of Plasma Science and Nuclear Fusion Research

Keywords: electron Bernstein wave (EBW), mode conversion, backward wave, interferometry, RF wave field measurement, overdense plasma

DOI: $10.1585 /$ pfr.3.013

Electron Bernstein Wave (EBW) heating is one of the most promising methods for high beta plasma heating. Electromagnetic waves in the Electron Cyclotron Range of Frequency (ECRF) have a cut-off density, whereas EBW has no density limit, and is strongly damped due to cyclotron damping. Since the EBW is an electrostatic wave with a short wavelength comparable to electron Larmor radius, it is necessary to excite this wave in plasma via the mode conversion process.

In linear devices, the electromagnetic field in the ECRF was investigated [1], and mode-converted EBW through X-mode injection from low field side was observed. Recently, electron cyclotron emission (ECE) was measured to evaluate the mode conversion efficiency [2,3].

The concept for confining high-beta plasma with an internal coil device is based on the theory of self-organized states in flowing plasma $[4,5]$. To achieve this concept, the production of overdense plasma (plasma density exceeds O-mode cutoff density) is expected by using a modeconverted EBW heating.

This study reports the preliminary results of ECRF electric field measurements in the internal coil device, Mini-RT [6]. We produce plasma by injecting an X-mode microwave $(2.45 \mathrm{GHz}, 2.5 \mathrm{~kW}$ c.w.). Figure 1 shows the typical magnetic configuration and measurement system. A monopole antenna inserted into the plasma detects the DC voltage signal, which is expressed as

author's e-mail: yatsuka@ppl.k.u-tokyo.ac.jp
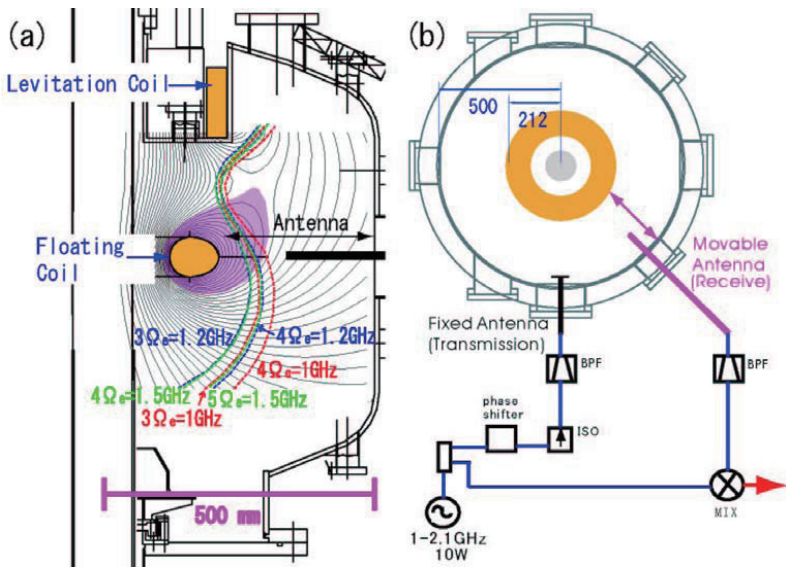

Fig. 1 (a) Cross section of the Mini-RT device. Solid (Dotted) lines denote magnetic surfaces (magnetic field strengths). (b) Top view of the Mini-RT, and block diagram of circuit.

$$
E_{0}(\boldsymbol{r})+E_{1}(\boldsymbol{r}) \cos \left[\phi(\boldsymbol{r})+\phi_{\mathrm{ph}}\right]
$$

where $E_{0}(\boldsymbol{r}), E_{1}(\boldsymbol{r})$, and $\phi(\boldsymbol{r})$ represent offset level, amplitude of ECRF electric field, and its phase, respectively. An external phase shift $\phi_{\mathrm{ph}}$ is controllable by a phase shifter. By measuring the electric field given by Eq. (1) for different external phase shifts, we can evaluate the parameters, $E_{0}(\boldsymbol{r}), E_{1}(\boldsymbol{r})$, and $\phi(\boldsymbol{r})$. We inject an additional microwave with an X-mode $(1-2.1 \mathrm{GHz}, 10 \mathrm{~W})$ into the overdense plasma (typical peak density is $7 \times 10^{16} \mathrm{~m}^{-3}$ ) using a 
ceramic-coated dipole antenna (element length is $100 \mathrm{~mm}$ ). The spatial profiles of ECRF electric fields for this microwave are measured using a coaxially-fed monopole antenna, which is $\sim 5 \mathrm{~mm}$ in length and is arranged to measure a toroidal component. A Band Pass Filter $(-44.5 \mathrm{~dB}$ @ $2.45 \mathrm{GHz}$ ) is set into the circuit to avoid a penetration of a high power-microwave $(2.45 \mathrm{GHz})$.

The radial profile of the refractive index is calculated by solving the dispersion relation with finite Larmor radius effect. As shown in Fig. 2, the mode conversion is expected to occur between the third and fourth harmonics of ECR.

Figure 3 shows the toroidal component of an ECRF electric field on the mid-plane. We can produce a plasma with a steep density gradient (e.g., $L_{\mathrm{n}} \sim 5 \mathrm{~cm}$ ) by introducing a separatrix configuration with a combination of float-

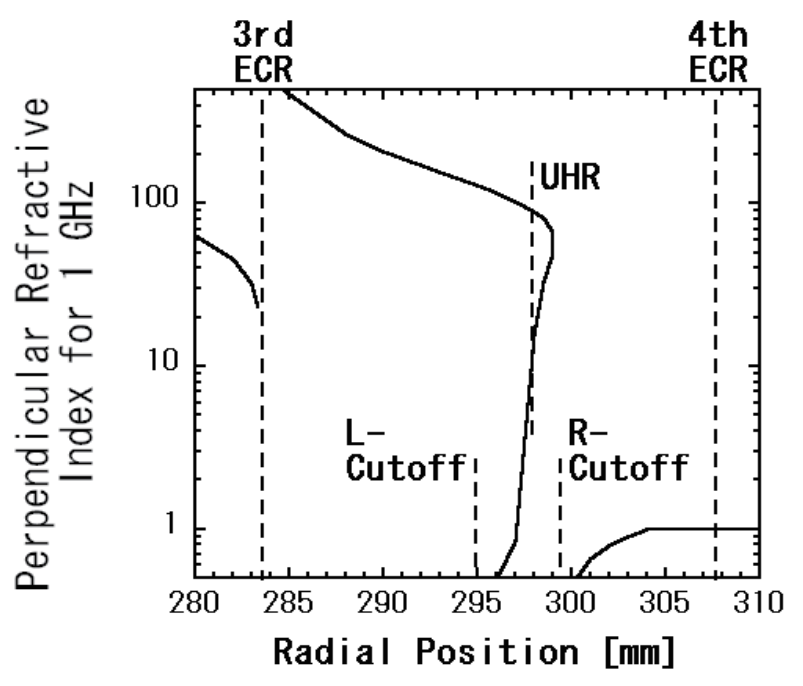

Fig. 2 Radial profile of refractive index for $1.0 \mathrm{GHz}$.

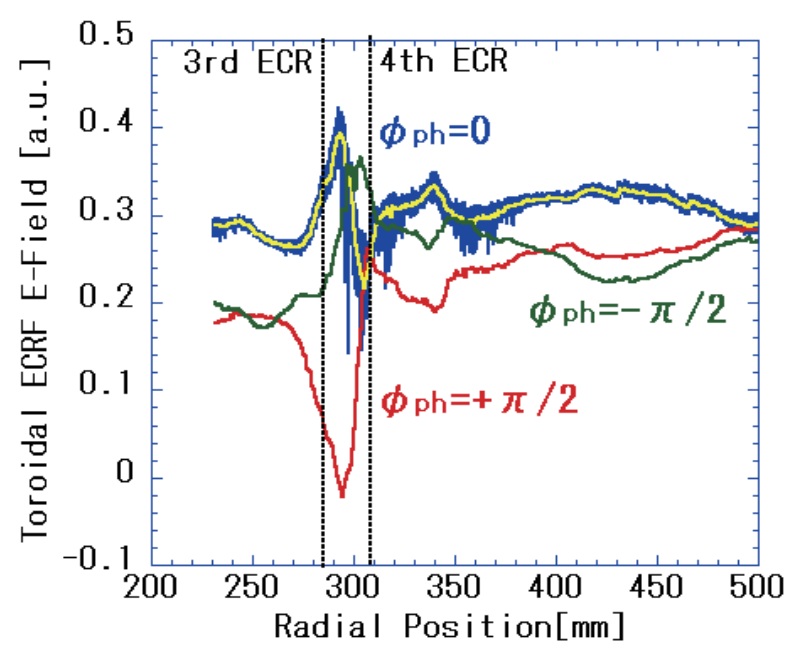

Fig. 3 Interferometer traces for $\phi_{\mathrm{ph}}= \pm \pi / 2$, 0 . Frequency of a launched wave was $1.0 \mathrm{GHz}$. We can obtain fluctuated data, and signals are averaged over each millimeter to determine amplitude and phase of an ECRF electric field. ing and levitation coils [7,8]. We can see a characteristic of short wavelength related with the EBW around major radius $R=310 \mathrm{~mm}$ in Fig. 3. The wavelength is evaluated to be $\sim 2 \mathrm{~cm}$ near the Upper Hybrid Resonance (UHR), which corresponds to the refractive index of $\sim 10$, whereas that in vacuum is considerably long (e.g., $\sim 30 \mathrm{~cm}$ ).

As shown in Fig. 3, radial electric fields are measured for three different phases to evaluate the phase of ECRF electric field. Radial profiles of the phases are shown in Fig. 4, and we have confirmed the phase reversal around the UHR. Similar experiments have been conducted for different frequencies, and the characteristic of phase reversal has been observed in all cases for each frequency. Results also indicate that this phenomenon occurs on the high-field side of the cyclotron harmonic resonance layer of each frequency, and the location coincides with a shortwavelength region. Around UHR, there are two modes, i.e., slow X-wave and EBW. A slow X-wave, which is all- (non-) reflective at L-cutoff (UHR), would represent a characteristic of a standing wave, where a phase is constant between the nodes, whereas the experimentally observed phases (Fig. 4) change gradually around the UHR. This might indicate a propagating wave, such as a modeconverted EBW.

Here, we consider the direction of group velocity, i.e., energy flux, which should be inward of the device. As shown in Fig. 4, a positive (negative) gradient of relative phase with respect to major radius indicates that a phase velocity is outward (inward) of the device. Therefore, this would suggest that the direction of phase velocity $v_{\mathrm{ph}}$ is opposite to group velocity $v_{\mathrm{g}}$ in this phase reversal region. Since the EBW has characteristics of a backward wave

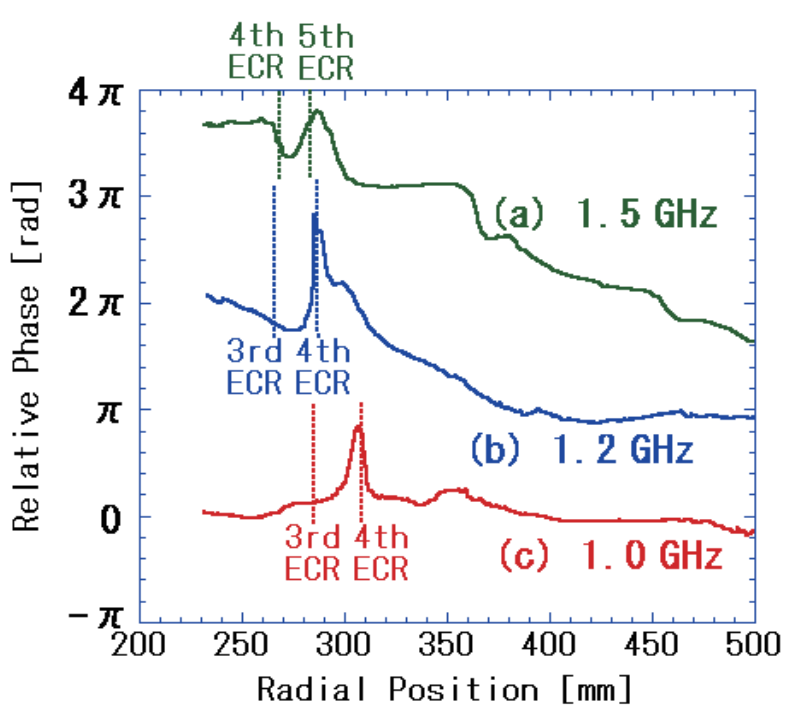

Fig. 4 Radial profiles of phase of an ECRF electric field with several frequencies. Dotted lines represent the locations of cyclotron harmonic resonance layers on mid-plane for each frequency. 
with a short wavelength [9], experimental results shown in Figs. 3 and 4 suggest the direct measurement of the mode-converted EBW between cyclotron harmonic resonance surfaces.

The authors would like to thank Prof. T. Kaneko for his technical advice on measurements. The authors also would like to thank Profs. T. Mito and N. Yanagi for their discussions about cooling and excitation of superconducting magnets.
[1] H. Sugai, Phys. Rev. Lett. 47, 1899 (1981).

[2] G. Taylor et al., Phys. Plasmas 9, 167 (2002).

[3] B. Jones et al., Phys. Rev. Lett. 90, 165001 (2003).

[4] S.M. Mahajan and Z. Yoshida, Phys. Rev. Lett. 81, 4863 (1998).

[5] Z. Yoshida et al., Plasma Fusion Res. 1, 008 (2006).

[6] Y. Ogawa et al., J. Plasma Fusion Res. 79, 643 (2003).

[7] T. Goto et al., Jpn. J. Apl. Phys. 45, 5197 (2006).

[8] E. Yatsuka et al., Trans. Fusion Sci. Tech. 51, 310 (2007).

[9] F.W. Crawford et al., J. Geophys. Res. 72, 57 (1967). 